(Aus dem pathologisch-physiologischen Institut der Stadt Köln.)

\title{
Über die Koeffizienten, die im Verein mit Koronararterienverschluss Herzkammerflimmern bewirken.
}

Von

Prof. H. E. Hering (Köln).

Der Zusammenhang zwischen Koronararterienverschluss (Kav) und Herzkammerflimmern (Hkf) wird in der Literatur zumeist mit der von Tigerstedt sowie Langendorff vertretenen Annahme erklärt, dass das Hkf bei der Unterbindung oder Abklemmung der Koronararterien durch Nebenverletzungen bewirkt wird.

Diese Nebenverletzungshypothese; wie ich sie nennen will, findet man immer wieder angeführt, und da sie sich an die Namen so angesehener Physiologen knüpft, ist es begreiflich, dass wenigstens viele von denjenigen, die sich mit den Folgen des Kav nicht selbst experimentell beschäftigt haben, geneigt sind, jene Hypothese anzunehmen oder wenigstens für die wahrscheinlichste zu halten. Man lese, um nur einige Beispiele aus der letzten Zeit anzuführen, was F. B. Hofmann ${ }^{1}$ ) in seiner vorzüglichen allgemeinen Physiologie des Herzens vom Jahre 1905 auf S. 240 darüber anführt, oder folgende Äusserung von L. Haberland ${ }^{2}$ ):

„Aus diesen Ergebnissen seiner Untersuchungen schliesst Langendorff wohl mit Recht, dass es bei den Versuchen mit Unterbindung der Koronararterie nicht die Herzanämie war, die das Flimmern hervorrief, sondern dass dafür jedenfalls $\mathrm{N}$ ebenverletzungen, die als mechanische Reize wirkten, verantwortlich gemacht werden müssen."

Das genauere Studium der einschlägigen Mitteilungen von Tigerstedt ${ }^{3}$ ) und Langendorff ${ }^{4}$ ) hat mir ergeben, dass nur

1) Nage l, Physiologie des Menschen Bd. 1. 1905.

2) Sammlung anat. u. physiol. Vorträge u. Aufsätze H. 26. 1914.

3) Skandinav. Arch. f. Physiol. Bd. 5 S. 71. 1893. - Physiol. Zentralbl, Bd. $9 \mathrm{Nr}, 18,1896$.

4) Pflüger's Arch. Bd. 61 S. 320. 1895.

Pflagger's Arehiv für Physiologie. Bd. 163. 
Langend orff und auch dieser nur einen einzigen eigenen Versuch über die Abklemmung einer Koronararterie anführt, auf den ich später noch zurückkomme. So gehören demnach auch diese Autoren zu jenen, die sich selbst mit den Folgen des Kav experimentell nicht oder so gut wie nicht beschäftigt haben. Dieser Umstand lässt mich die Aufstellung und das Festhalten der Nebenverletzungshypothese dieser Autoren wenigstens zum Teil verstehen. Zum anderen Teil ist die Aufstellung jener Hypothese dadurch zu erklären, dass jene Autoren gegen die Annahme waren, das Hkf dureh Kav beruhe auf Anämie; so entstand schon aus der Stellungnahme gegen die Anämiehypothese das Bedürfnis nach einer anderen Hypothese. -

Es war auf dem X. Kongress für innere Medizin im Jahre 1891, als. Frey ${ }^{1}$ ) unter Bezugnahme auf die Cohnbeim'schen ${ }^{2}$ ) Versuche unter anderem meinte, „dass die Anämie des Herzmuskels nach den vorliegenden physiologischen Erfahrungen eine relativ ungefährliche Erschcinung ist."

K. Tigerstedt kam dann 1893 in seiner Mitteilung zu dem Schluss: "Dass derjenigo Herzstillstand, den Cohnheim und v. Schulthess-Rechberg beobachtet haben, nicht durch die Anämie eines umschriebenen Teiles der Herzwand, sondern durch Nebenverletzungen bedingt ist."

Hier finde ich zum ersten Male den Ausdruck "Nebenverletzungen".

O. Langend orff schloss sich 1895 der Nebenverletzungshypothese an. Auf S. 324 kommt er zu folgendem Schluss: "Diese meine Ergebnisse stehen in vollständigem Einklang mit der Meinung derjenigen, die wie v. Frey, Tigerstedt u. a. den dauend schädigenden Einfluss der vorübergehenden Aufhebung der Blutzufuhr zum Herzmuskel leugnen und den Grund für die von v. Bezold, Cohnheim usw. erhaltenen Resultate auf $\mathrm{Neben-}$ verletzungen beziehen."

Langendorff führt auf $\mathrm{S}$. 321 ausserdem an, dass schon Newell Martin und Sedgwick (1882) sowie auch Fenoglio und Drogoul (1888) auf die Möglichkeit hingewiesen haben, dass mit der Ligatur der Kranzgefässe verbundene mechanische Alte-

1) Verhandl. d. Kongr. f. innere Med. 1891 S. 277.

2) Virchow's Arch. Bd. 85 S. 503. 1881. 
rationen benachbarter Teile schuld an den beobachteten Störungen sein können.

Gegenüber dieser Nebenverletzungshypothese hielt Porter ${ }^{1}$ ) an der schon von Cohnheim und v. Schulthess geäusserten Anämiehypothese fest. -

Bei der Differenz der Meinungen über das Entstehen des Hkf nach Kav würde man erwarten, in der Literatur Mitteilungen zu finden, die sich speziell experimentell mit dem Hkf nach Kav befassen; das ist aber nicht der Fall und wohl mitbedingt dadurch, dass man die Bedeutung des Flimmerns zur Frklärung pathologischer Erscheinungen beim Menschen noch nicht zu würdigen wusste. Über die Folgen des Kav ist zwar sehr viel experimentiert worden; die Absichten, mit denen man an diese Experimente heranging, waren jedoch sehr verschieden, und eigentlich beschäftigt sich experimentell nur die Mitteilung von Porter im Jahre 1896 mit dem Hkf nach Kav, nachdem Tigersted t, wie Porter sich ausdrückt, „versucht, den ganzen Streit auf das Vorbandensein dieses Flimmerns jetzt zuzuspitzen". In dieser Mitteilung sagt Porter, dass das Flimmern ein allgemein bekanntes Glied des gesamten Symptomenkomplexes nach Verschluss der Koronararterien ist; es geht aber aus seiner Mitteilung nicht hervor, dass er erkannt hătte, dass der "Stillstand des Herzens" durch das Flimmern bedingt ist, oder mit anderen Worten, dass das Flimmern und nicht der "Stillstand des Herzens" das Primäre ist. Aus S. 131 seiner früheren Mitteilung ${ }^{2}$ ) ergibt sich, dass Porter tatsächlich meint, der Stillstand ginge dem Flimmern voraus; von zehn Fällen, gibt er auf S. 131 an, folgte in sechs das Flimmern dem Stillstand instantly", in vier nach $2-3$ Sekunden. In der Kurve der Fig. 1 seiner Mitteilung sieht man aber nichts vom "Stillstand".

Der Ausdruck "Stillstand des Herzens" ist hier überhaupt nicht angebracht, denn das Herz bzw. die Kammern stehen unter den genannten Umständen nicht still, sondern sie flimmern. Dem wird Porter insofern gerecht, als er mehrfach statt nur vom "Stillstand" zu reden, vom "Stillstand des Herzens mit fibrillären Zuckungen" oder "Stillstand mit Flimmern" spricht. Wer sich nun nicht selbst experimentell mit den Folgen des Kav beschäftigt hat, könnte dem-

1) Physiol. Zentralbl. Bd. 9 Nr. 22. 1896.

2) Journ. of Physiol. vol. 15 no. 3. 1893. 
nach glauben, und es ist, wie die Literatur lehrt, auch geglaubt worden, das. Primäre wäre der Stillstand des Herzens, an dẹ sich erst das Flimmern anschliesst, während nach meiner Erfahrung das Primäre das Hkf ist, und das Herz erst einige Zeit nachber mit Aufhören des Flimmerns wirklich zum Stillstand kommt ${ }^{1}$ ).

Den Ausdruck "Herzstillstand" als Folge des Kav haben schon Cohnheim und v. Schulthess-Rechberg gebraucht, nach meiner Meinung dort, wo sie von ihm sprechen, mit Unrecht. Sie gaben an, dass nach Verschluss eines der grossen Koronaräste die Kammern erst stillstehen und dann flimmerten, während ich dies nach Kav nie gesehen babe, obwohl ich die Tätigkeit der Kammern immer graphisch verzeichnet habe. Aller Wahrscheinlichkeit nach ist es ihnen, wie auch Porter, entgangen, dass die Kammern zur Zeit des anseheinenden Stillstandes schon flimmerten. So kam es auch, dass sie, obwohl sie das Flimmern, nachdem es dann grobschlägiger geworden war, sehr wohl beobachtet haben, nicht erkanten, dass das Wesentliche und Primäre des "plötzlichen Herzstillstandes" nach Koronararterienverschluss das Herzkammerflimmern ist.

Jene unzutreffende Beschreibung in der Aufeinanderfolge der Erscheinungen hatte zum Beispiel zur Folge, dass Vier ordt 1891 die Erklärung des fluttering heart beim Menschen durch die Experimente Cohnheim's ablehnte, da nach diesen die Erscheinungen derart verlaufen, dass das Herz „in beiden Ventrikeln plötzlich stillsteht, und dass $d$ ann nach einer gewissen Pause jene wühlenden Bewegungen auftreten".

Abgesehen davon, dass ich einen "plötzlichen Herzstillstand“ nach Kav trotz graphiseher Aufnabme noch nie gesehen habe, wäre nach meiner zwanzigjährigen sehr reicblichen Erfahrung über das Verhalten des. Säugetierherzens ein solch plötzlich einsetzender wirklicher Stillstand der Kammern nach Kav nur verständlich durch Aufhebung der Überleitung von den Vorhöfen zu den Kammern.

1) Porter gibt zwar an, dass es ihm in einem einzigen Falle nach Verschluss der linken Koronararterie unmöglich war, die gewöhnlichen fibrillären Zuckungen festzustellen; das Herz scheint aber doch, wenn auch nicht „ausgesprochen", geflimmert zu haben, da er zum Schluss schreibt: „Das Herz kam in diesem Falle sehr langsam zum Stillstande, und die Erregbarkeit der Muskelfasern war dabei wahrscheinlich zu sehr herabgesetzt, um ein ausgesprochenes Flimmern zu erlauben." 
Dass es auf diese Weise nach Kav zunächst zum Stillstand der Kammern und dann erst anschliessend daran zum Flimmern der letzteren kommen könnte, ist wohl möglich, nach Kav habe ich jedoch etwas derartiges bis jetzt noch nie beobachtet. -

Wenn wir nun statt "Herzstillstand" Hkf setzen, so hat schon v. Frey ${ }^{1}$, sich stützend auf die Versuche von Fenoglio und Drogoul sowie auf die von Porter aus dem Jahre 1893, im Jahre 1894 gesagt :

„Aus diesen Versuchen folgt, dass der Herzstillstand nicht eine notwendige Folge der Verschliessung grosser Koronaräste darstellt." Sofern wir, wie gesagt, statt Herzstillstand Hkf setzen, ist diese Folgerung ganz richtig.

"Entweder", setzt v. Frey fort, „muss zur Anämie des abgesperrten Herzteiles noch etwas hinzukommen, wodurch der Stillstand herbeigeführt wird, oder es ist neben der Grösse auch der Ort der Schädigung von besonderer Bedeutung." Wir werden auf diese Punkte noch zurückkommen. An dieser Stelle sei nur hervorgehoben, dass v. Frey nach dieser Äusserung auf dem richtigen Wege war, um den Zusammenhang zwischen Kav und Hkf zu aralysieren. Unter dem richtigen Wege verstehe ich hier die Anwendung der durchaus nicht neuen, aber immer wieder vernachlässigten Erkenntnis, dass ein Geschehen nicht nur durch eine, sondern durch mehrere Ursachen oder Koeffizienten ${ }^{2}$ ) zustande kommt. Ein jeder Eingriff am Organismus, wie zum Beispiel der Kav, ist nur ein Koeffizient, der erst im Verein mit anderen Koeffizienten ein bestimmtes Geschehen, hier das Hkf bewirkt. Ohne letztere tritt das Hkf nicht auf, und insofern ist es auch keine notwendige Folge des Kav. So ist auch das Hkf nicht der einzige Effekt des Kav, vielmehr hat dieser eine ganze Anzahl Koeffekte im Gefolge. Von diesen ist in der vorliegenden Mitteilung unser Interesse speziell einem Koeffekt zugewendet, dem Hkf.

Im folgenden wollen wir nun versuchen, soweit dies auf Grund eigener Experimente und unter Zuhilfenahme der Literatur his jetzt möglich ist, folgende Frage zu beantworten: Welches sind die Koeffizienten, die im Verein mit Koronararterienverschluss Herzkammerflimmern bewirken?

1) Zeitschr. f. klin. Med. Bd. 25. 1894.

2) Die Naturwissenschaften H. 7. 1913. 
Wir dürfen nicht erwarten, auf diese Frage heute schon eine vollkommen befriedigende Antwort zu bekommen, denn es bedarf hierzu noch zahlreicher experimenteller Untersuchungen, wohl aber hoffen, auf diesem Wege zu einer grösseren Klarbeit zu gelangen.

Die folgenden Darlegungen gelten zunächst alle für den akuten Verschluss einer oder mehrerer Koronararterien, wie er experimentell durch Ligatur, Abklemmung oder Verstopfung von innen aus bewirkt werden kann; sie beziehen sich, wenn nichts anderes gesagt ist, auf Experimente an Hunden. Meine Versuche reichen bis zum Jahre 1909 zurück und erstrecken sich auf 20 Hunde; sechs Versuche machte ich hier im Institute mit Dr. Beck und Dr. Kisch im Juli 1914, bis der Krieg die Fortsetzung der Experimente unterbrach.

\section{Die Grösse der versehlossenen Koronararterie.}

Dass die Grösse der verschlossenen Koronararterie eine Rolle spielt, ob der Versehluss Hkf zur Folge hat oder nicht, das lässt sich schon der Mitteilung von Cohnheim und v. SchulthessRechberg entnehmen, wenn wir, wie schon weiter oben erwähnt, an Stelle von Herzstillstand Kammerflimmern setzen. Die Autoren wussten sehr wohl, „dass nach dem Verschlusse kleinerer Arterienäste nicht jedesmal die viel geschilderte Erscheinungsreihe eintritt “, und hielten es nur für genügend festgestellt, „dass die geschilderte Folge von Erscheinungen die direkte und ausschliessliche Wirkung des Verschlusses mindestens e in es grösseren Kranzarterienzweiges ist." Mit der Grösse der verschlossenen Arterie sich zu befassen, veranlasste sie besonders folgende Überlegung:

„Wenn wirklich in dem akut anämisch gemachten Bezirk des Herzmuskels ein Herzgift sich entwickelt, so sollte man meinen, dass die Geschwindigkeit, mit welcher die zur vollen Wirksamkeit erforderliche Quantität derselben sich bildet, und die Grösse des ausgeschalteten Bezirkes in gleichem Verhältnis zunimmt. " Aus ihren, zu diesem Zwecke angestellten Versuchen schlossen sie, „dass die Vergrösserung des akut anämisierten Bezirkes über einen gewissen Umfang hinaus den Eintritt des Herzstillstandes nicht mehr beschleunigt", und ferner, „dass die Verkleinerung desselben unter eine gewisse Grösse den Erfolg des Eingriffes überhaupt unsicher macht oder ihn selbst völlig ausschliesst. Innerhalb dieser Grenzen, nach oben und unten, aber scheint in der Tat die Geschwindigkeit des Eintritts der Wirkung in gleichem Verhältnis mit der Grösse des seiner arteriellen Zufubr beraubten Bezirkes zu erwachsen."

Zunächst sei bemerkt, dass die Autoren hier an Stelle der Grösse der Arterie die Grösse des anämisierten Bezirkes setzen. 
Dies ist vom ihrem Standpunkt aus wohl richtig, da nach ihrer Meinung „die Äste der Koronararterien keinerlei Anastomosen miteinander eingehen".

Heute wissen wir, dass diese Meinung nicht zutreffend ist, vielmehr das Herz, wie sich Spalteholz ${ }^{1}$ ) ausdrückt, ausserordentlich reich an arteriellen Anastomosen ist. Trotzdem wird es im allgemeinen wohl richtig sein, dass mit der Grösse der verschlossenen Arterie anch die Grösse des Bezirkes zunimmt, der durch die lokale Behinderung des arteriellen Zuflusses betroffen wird.

Wenn wir in diesem Abschnitte die Grösse der Arterie und nicht die Grösse des betroffenen Bezirkes in den Vordergrund stellen, so gesehieht dies, weil das Sirhere die Grösse der Arterie ist und man sich über die Grösse des betroffenen Bezirkes schwieriger Aufschluss versebaffen kann mit Ausnahme jenen Bezirkes, der an der Aussenoberflache des Herzens leicht sichtbar ist (s. weiter unten).

Cohnheim und v. Schulthess-Rechberg kamen, wie erwähnt, zu dem Schluss, dass die Geschwindigkeit des Eintrittes der Wirkung innerhalb gewisser Grenzen in gleichem Verhältnis mit der Grösse des seiner arteriellen Zufuhr beraubten Bezirkes wachse. Sagen wir nun statt Grösse des Bezirkes Grösse der Arterie, um die es sich bei ibren Feststellungen in Wirklichkeit bandelte, so stimmt jener Satz nicht ohne weiteres mit folgenden von mir und anderen gemachten Erfahrungen: Das Intervall zwischen Koronar. arterienverschluss und Auftreten des Herzkammerflimmerns kann nach Verschluss der rechten Koronararterie sehr verschieden gross sein.

Ich habe mich speziell mit Rücksicht auf die Nebenverletzungshypothese für das Intervall Kav-Hfk interessiert und es an der Coronaria dextra studiert; das kürzeste betrug 1 Min. 30 Sek., das längste 45 Min.; dazwischen 2 Min. 30 Sek., (zweimal) 2 Min. 45 Sek., 10 Min., 17 Min., 20 Min., 33 Min. 35 Sek.

Nach Cohnheim und v. Schulthess-Rechberg dauerte es vom. Verschluss der Coronaria dextra bis zum tötlichen Herzstillstand mindestens 5 Minuten.

Porter verschloss die A. cor. dextr. 14 mal und beobachtete nur 2 mal Stillstand; nach seiner ersten Mitteilung einmal nach 108 Sekunden; für das zweite Mal fehlt die Zeitangabe.

1) Deutsch. med. Wochensshr. $1907 \mathrm{Nr} .20$. 
Aus einer Tabelle der Versuche, die Thomas Lew is ${ }^{1}$ ) zum Zwecke des Studiums der paroxysmalen Tachykardie vornahm, geht hervor, dass er zwölf Versuche mit Ligatur der Coron. dextr. machte. Vier Versuche brach er ab; in den übrigen acht Versuchen beobachtete er Kammerflimmern nach 57 Min., 1 Stde. 12 Min., 1 Stde. 25 Min., 1 Stde. 45 Min., 1 Stde. 46 Min., 1 Stde. 56 Min., 2 Stdn. 12 Min., 3 Stdn. 13 Min.

Wenn auch die Grösse der rechten Koronararterie etwas variiert, die Grösse ihres Versorgungsgebiets infolge Variation der Kollateralen etwas verschieden sein und die Arterie nicht inmer unmittelbar an der Aorta unterbunden sein wird (was nötig ist, da bald nach ibrem Ursprung Ästchen von der Coron. dextr. abgehen), so können doch diese auf die Gefässe sich beziehenden Variationen die grosse Verschiedenheit des Intervalls nicht erklären.

Mit ihrem ohen erwähnten Satze hielten Cohnheim und v. Schulthess-Rechberg in gutem Einliange:

„Dass nach dem Verschluss der Coron. dextra bis zum Eintreten der Unregelmässigkeit der Schlagfolge und dem Herzstillstand eine nicht unerheblich längere Zeit zu vergehen pflegt als nach Ligatur der ungleich stärkeren grossen Äste der Coron. sinistra."

Wälirend es nach ihren Erfahrungen vom Verschluss der Coron. dextra bis zum tötlichen Herzstillstand, wie schon erwähnt, mindestens 5 Minuten dauerte, betrug diese Zeit nach Ligatur einer der grossen Äste der Coron. sinistra 2 Minuten im Maximum.

Nicht nach dem Intervall, sondern danach, ob überhaupt „Stillstand" des Herzens eintrat, beurteilte Porter die Folgen des Verschlusses verschiedener Arterien des Herzens. Er stellte folgende Tabelle auf:

\begin{tabular}{|c|c|c|c|}
\hline Arterie & Verschluss & Stillstand & Prozent \\
\hline Stamm der A. coron. sin. & . $19 \mathrm{mal}$ & $19 \mathrm{mal}$ & 100 \\
\hline Ramus circumflexus . & . 11 & 7 & 64 \\
\hline Ramus descendens . & 39 & $11 \%$ & 28 \\
\hline A. coron. dextra. . & 14 & $2 \%$ & 14 \\
\hline R. septi der A. coron. si & 3 & $\ldots$ & \\
\hline
\end{tabular}

Aus diesen Ergebnissen schloss Porter: „Die Häufigkeit des Stillstandes des Herzens steht also im Verbältnisse zu der Grösse der verschlossenen Arterien."

1) Heart vol. 1 S. 98.1909 . 
Während Cohiheim und vo Schulthess-Rechberg bei dem Vergleich der Ergebnisse der an verschiedenen Arterien angestellten Versuche aber nur die Grösse der Arterie in Betracht zogen, fügte Porter hinzu, "dass der Zustand des Herzens zur Zeit der Verschliessung auch von Einfluss ist".

Auf diesen sehr wichtigen Koeffizienten, den Zustand des Herzens, auf den wir weiter unten noch ausführlicher zurückkommen werden, ist es jedenfalls mit zurückzuführen, dass das Intervall $\mathrm{Kav}-\mathrm{Hkf}$ bei Verschluss derselben Arterie an verschiedenen Herzen so verschieden gross sein kann.

Aus der Gesamtheit der vorliegenden Erfahrungen ist wohl zu ersehen, dass die Grösse der verschlossenen Koronararterie eine Rolle spielt, indem unter sonst ungefähr gleichen Umständen die Wabrscheinlichkeit des Auftretens von Hkf mit der Grösse der verschlossenen Koronararterie wächst, dass es aber bei Verschluss derselben Arterie an verschiedenen Herzen sehr auf seinen Zustand ankommt, ob überhaupt Hkf eintritt und wie rasch es nach dem Kav erscheint.

Einen Koeffizienten sehen wir aber weder bei Cohnheim und v. Schulthess-Rechberg noch bei Porter berücksichtigt, das ist die Funktion des betroffenen Herzbezirkes; es ist jener Koeffizient, den v. Frey vielleicht meinte, als er von dem "Ort der Schädigung" sprach, und den wir besprechen wollen, bevor wir die Bedeutung des Zustandes des Herzens weiter erörtern.

\section{Die Funktion des von der Koronararterie versorgten Herzbezirkes.}

Dass man sich früher mit der eventuellen Bedeutung der Funktion des betroffenen Herzbezirkes für das Hkf nach Kav nicht weiter beschäftigt hat, ist insofern begreiflich, als wan dazu keine genügende Veranlassung hatte. Porter ${ }^{1}$ ) hat zwar gerade mit Hilfe des Kav „Über die Frage eines Koordinationszentrums im Herzventrikel" 1893 eine Untersuchung angestellt; da er aber zu dem Ergebnis kam, „dass kein Koordinationszentrum im gewöhnlichen Sinne einer begrenzten Zusammenhäufung der Nervenzellen besteht, sei es im Septum, sei es in irgend einem anderen Teil der Ventrikularwand," hatte er keinen Anlass, die Funktion des be-

1) Pflüger's Arch. Bd. 55. 1893. 
troffenen Herzbezirkes zur Erklärung der verschiedenen Ergebnisse nach Verschluss der verschiedenen Arterien zur Erklärung mit heranzuziehen.

Seit wir nun über das Reizleitungssystem und seine Verteilung in den Kammern so gut unterrichtet sind, hat die Frage nach der Örtlichkeit des betroffenen Bezirkes eine besondere Bedeutung bekommen, ohne dass speziell von diesem Gesichtspunkte aus Versuche uber die Folgen des Verschlusses verschiedener Koronararterien gemacht worden wären.

Ich habe daher zu diesem Zwecke im Wintersemester 1909-1910 eine Anzahl solcher Versuche an Hunden ausgeführt. Ich unterband die rechte oder Äste der linken Koronararterie und wartete den Erfolg. der Unterbindung ab. Nach Beendigung des Versuches durehströmte ich von der Aorta aus die Herzen mit Neutralrot ${ }^{1}$ ), wodurch sich der infolge der Unterbindung nicht gefärbte Herzbezirk gut von dem übrigen gefärbten Herzen abhob. Die Herzen wurden dann zur anatomischen Untersuchung konserviert. Einige dieser Herzen brachte ich im April 1910 zur Erlanger Tagung der Patbologischen Gesellschaft mit und übergab sie Kollegen A sch off zur anatomischen Untersuchung, zu der er jedoch nicht kam.

Das Ergebnis meiner damaligen Versuche ging dahin, dass ich aus ihnen keine sicheren Anhaltspunkte dafür entnehmen konnte, dass die Örtlichkeit des betroffenen Bezirkes eine entschiedene Rolle spiele.

Im Jahre 1911 hat dann G. Haas ${ }^{2}$ ) eine bei Asch off angeführte Arbeit „Über die Gefässversorgung des Reizleitungssystems des Herzens" veröffentlicht, in der er sich auch mit dem Hundeherzen beschäftigt und dazu auf S. 654 bemerkt: „,dass die Wirkungen von Unterbindungen am Kranzgefässe am Hundeherzen nur richtig beurteilt werden können, wenn man weiss, dass gerade beim Hunde nicht die rechte Kranzarterie wie beim Menschen, sondern die linke die Hauptabschnitte des Reizleitungssystems speist".

Dazu liegen nun folgende Erfahrungen von mir vor:

In zwölf Versuchen beim Hunde sah ich nach Ligatur der Coron. dextra zehnmal Kammerflimmern auftreten; in den zwei ubrigen Fällen wurde nach 28 Min. bzw.

1) Siehe auch Verhandl. d. Kongr. f. innere Med. 1907 S. 581.

2) Anat. Hefte Bd. 43 H. 131. 1911. 
40 Min. die Ligatur des R. descendens der linken Coronaria angeschlossen, worauf nach 1 Min. bzw. 16 Min. die Kammern flimmerten。

Das Intervall Kav-Hkf, das ich schon weiter oben angeführt habe, war gerade in den letzten Versuchen, die ich mit Dr. Beck und Dr. K isch ausführte, in vier Fällen besonders kurz: 1 Min. 30 Sek., 2 Min. 30 Sek., 2 Min. 45 Sek., 2 Min. 45 Sek.

Diese Erfabrungen sind unter Zugrundelegung der anatomischen Angaben von G. Haas nicht geeignet, die Annahme zu stützen; dass das Hkf nach Verschluss der Coronaria dextra von den Hauptabschnitten des Reizleitungssystems, worunter $\mathrm{Haas}$ den Atrioventrikularknoten und den Bündelstamm versteht, ausgeht.

Damit ist aber nicht gesagt, dass das nach Verschluss der Coronaria dextra auftretende Hkf überhaupt nicht vom Reizleitungssystem ausgeht, denn seine Endausbreitungen finden sich nach T a w a $\mathrm{a}^{1}$ ) subendokardial auch an der Wand der rechten Kammer, also im Hauptspeisungsgebiet der Coron. dextra.

Dass das Kammerflimmern nicht vom Atrioventrikularknoten inkl. eines Teiles des Bündelstammes ausgehen $\mathrm{muss}$, habe ich bei mechanischer oder elektrischer Reizung der verschiedensten Teile der Kammern oft genug beobachtet, wie auch; dass Teile der Kammern flimmerten, die weder das Bündel noch seine beiden Hauptschenkel enthielten. Es kann also das Kammerflimmern sicher auch von Teilen ausgehen, die nicht die Hauptabschnitte des Reizleitungssystems enthalten; demnach kann dies auch bei Hkf nach Kav der Fall sein.

Schliesst also einerseits der Befund von $\mathrm{Ha}$ as nicht aus, dass auch nach Verschluss der Coron. dextra das Hkf vom Reizleitungssystem ausgehen kana, so scheint er mir andererseits geeignet zu sein, zur Erklärung der Verschiedenheit der Ergebnisse nach Verschluss verschiedener Koronararterien beizutragen. Cohnheim und v. Schulthess-Rechberg sowie Porter haben, wie erwähnt, jene Verschiedenheit auf die verschiedene Grösse der Arterien, Porter auch auf den verschiedenen Zustand des Herzens mitbezogen, auf den wir noch zu sprechen kommen.

Vermutlich ist nun aber ausser diesen beiden Koeffizienten auch der Ort des betroffenen Bezirkes zur Erklärung mit heranzuziehen.

1) Das Reizleitungssystem des Säugetierherzens. Jena 1906. - Siehe auch J. G. Mönckeberg; Ergebn. d. allg. Path. u. path. Anat. 1910 S. 625 u. 637. 
Dafür spräche vorläufig folgendes. Das Intervall Kav-Hkf ist nach Verschluss der rechten Koronararterie, entsprechend den vorliegenden weiter oben erwähnten zahlreichen Angaben, in der weitaus grössten Zahl der bis jetzt vorliegenden Fälle sehr lang bzw. kommt es uberhaupt während der Beobachtungszeit nicht zum Hkf.

Nun ist zwar nach den Angaben von $\mathrm{Cohnh}$ eim und v. Schulthess-Rechberg die Coron. dextra „viel schwächer, als einer der beiden Hauptäste der Sinistra", das ist der R. circumflexus und der "nahezu ebenso starke" $R$. descendens; es ist aber andererseits, um hier nur die Angaben von Porter zugrunde zu legen, der Effekt des Verschlusses der untereinander nahezu gleich starken Hauptäste recht versehieden, indem nach Verschluss des R. circumflexus in $64 \%$, nach Verschluss des nahezu ebenso starken R. descendens nur in $28 \%$ der Fälle Hkf eintrat. Diese grosse Verschiedenheit könnte vielleicht mehr auf der Mitversorgung der Hauptsysteme des Reizleitungssystems durch den R. circumflexus, als auf der Grössenverschiedenheit der beiden Hauptäste beruhen und so sich auch der geringere Prozentsatz $(14 \%)$ der positiven Fälle nach Verschluss der A. coron. dextra in den Versuchen Porter's gegenüber dem so viel höheren Prozentsatz nach Verschluss des R. circumflexus erklären.

Bei der Zahl der in Betracht kommenden Koeffizienten, vou denen, wie wir im nächsten Abschnitt hören werden, der Zustand des Herzens von grösster Bedeutung ist, lässt sich auf Grund der bis jetzt vorliegenden Versuchsergebnisse über den Kav noch nicht mit Sicherheit sagen, dass die Örtlichkeit des betroffenen Bezirkes und damit seine Funktion eine wesentliche Rolle bei dem Auftreten von Hkf nach Kav spiele. Wenn ich trotzdem zu dieser Annahme neige, so unterstützt mich darin die Erfahrung, dass die Reizbildungsfähigkeit des Reizleitungssystems im allgemeinen um so grösser ist, je näher die heterotope Reizbildungsstelle der nomotopen liegt, worauf ich schon wiederholt aufmerksam gemacht habe. Danach wird das Hkf unter sonst gleichen Umständen leichter von den Hauptabschnitten des Reizleitungssystems der Kammern ausgehen als von seinen Ausläufern oder der übrigen Herzkammermuskulatur, womit sich die bis jetzt vorliegenden Ergebnisse über das Auftreten von Hkf mit Kav gut in Einklang bringen lassen. 
Bezüglich der Gefässversorgung des Hundeherzens bemerkt G. Haas noch folgendes: "Für Unterbindungsversuche, die im Effekt speziell den Knoten und das Atrioventrikularbündel treffen sollen, ist es wichtig; nicht nur den Ramus septi fibrosi coronariae sinistrae auszuschalten, sondern auch die grosse Septumarterie. Sie geht unmittelbar aus der Mündungsstelle der linken Koronararterie hervor, gelegentlich auch aus einem gesonderten Ostium, das dann im untersten Teil der Aortentasche zu suchen ist."

Hierzu sei eine Stelle der S. 511 ans der Mitteilung von Cohnheim und v. Schulthess-Rechberg erwähnt:

"Ganz oben, bald nach seinem Ursprunge, und zuweilen sogar so hoch oben, dass eigentlich eine Dreiteilung des Stammes der linken Kranzarterie vorliegt, entspringt aus ihm ein starker Ast, der sogleich ins Septum ventriculorum tritt und hier schräg von vorn oben nach hinten unten auf der dem rechten Ventrikel zugekehrten Seite desselben, nur von spärlichen Muskelbündeln bedeckt, verläuft; er gibt nach beiden Seiten zahlreiche $\ddot{A}$ ste ab. Eine Folge dieses hohen Ursprungs des R. septi war es, wie gleich hier bemerkt werden mag, dass dasselbe in allen unseren Versuchen nur ein einziges Mal mit abgebunden worden ist."

Der Ast, den die Autoren hier $R$. septi nennen, entspricht bei Haas wohl die Arteria septi fibrosi. Auch Porter kannte allem Anschein nach nur letztere und nennt sie auch R. septi. Demnach ist die "grosse Septumarterie" wohl noch nicht isoliert unterbunden worden, und es wird Gegenstand weiterer Versuche sein müssen, zu erfahren, welchen Effekt ihre Unterbindung hat.

Nach der weiter oben angeführten Tabelle beobachtete Porter nach Verschluss des R. septi in keinem der drei Fälle "Stillstand": des Herzens.

\section{Der Zustand der Herzkammern.}

Wir kommen jetzt zu einem Koeffizienten des Hkf, der eine sehr grosse Bedeutung hat, das ist der Zustand (Z) der Herzkammern. Schon von Me Willi a m ${ }^{1}$ ) wurde hervorgehoben, dass das Herz, je nach den jeweiligen Umständen, schwerer oder leichter ins Flimmern gerät, wovon ich mich bei vielen Experimenten oft genug überzeugt habe. Bevor man darangeht, die Abklemmung oder Abbindung einer Koronararterie vorzunehmen, ist schon durch die diesem Akte vorausgehenden Eingriffe der Zustand der Herzkammer gegenüber der Norm zweifellos etwas geändert, worden. Wie stark sich aber Z geändert hat und in welchem Sinne die einzelnen Fingriffe $Z$ geändert haben, ist noch wenig untersucht worden. Eine hierhergehörige Angabe rührt von $\mathrm{P}$ orter ${ }^{2}$ ) her und lautet folgendermassen :

1) Journ. of Physiol. vol. 8.

2) Pbysiol. Zentralbl. Bd. 9 Nr. 222. 1896. 
„Dass der Zustand des Herzens zur Zeit der Verschliessung auch von Einfluss ist, ersieht man aus den Folgen der Unterbindung des R. descendens bei 39 Hunden. 14 von diesen waren mit Morphium oder Curare oder beiden in gewöhnlicher Dosis vorbereitet, während 25 nur ätherisiert oder ätherisiert und durch Trennung der Medulla oblongata bewegungslos gemacht wurden. Unter den 14 erstgenannten wurde das Herz neunmal $(64 \%)$ zum Stillstand gebracht; unter den 25 letztgenannten, welche kein Morphium und Curare empfangen hatten, stand das Herz nur. zweimal (nur 26\%) still. Bei allen Tieren wurde die Arterie in der Nähe ihres Ursprunges unterbunden."

Mit diesen Angaben stimmt überein, dass nach unseren Erfahrungen im Institute dem Morphium eine fördernde Wirkung auf die heterotope Reizbildung zukommt ${ }^{1}$ ).

Es wäre ferner hier zu erwähncn, dass C. Hirsch ${ }^{2}$ ) nach Unterbindung des R. descendens bei acht Hunden und zwei Affen in keinem Falle "Herzstillstand" eintreten sah; er verwendete zur Narkose nur Äther ${ }^{3}$ ) und, um die Operation möglichst schonend vorzunehmen, das Überdruckverfahren von Brauer, wobei Sauerstoff in die Lungen eingeleitet wird.

Bei meinen Versuchen wurden die Hunde erst morphinisiert und dann noch mit Äther oder seltener mit einer Äther-Chloroformmischung narkotisiert, bis sie nach Thoraxeröffnung curaresiert und künstlich geatmet wurden.

In den erwähnten Experimenten von $T h$. Lewis waren die Hunde mit Morphium und Paraldehyd narkotisiert unter ergänzender Narkose mit Äther zur Erzielung einer kompletten Anästhesie.

Es ist schon länger bekannt und von mir bei Hunden und Katzen wiederholt beobachtet worden, dass die Tiere in der Narkose, besonders bei Anwendung von Chloroform, plötzlich an Hkf zugrunde gehen können. In der letzten Zeit hat sich Levy ${ }^{4}$ ) mit diesem Gegenstand eingehender befasst und festgestellt, dass bei Katzen das Herz besonders in leichter Chloroformnarkose häufig ins Flimmern gerät, während tiefe Chloroformnarkose das Auftreten von Flimmern zu verhindern vermag.

1) Zeitschr. f. exper. Path. u. Ther. Bd. 12 H. 3 S. 389. 1913.

2) Deutsch. med. Wochenschr. 1907 Nr. 20.

3) Bei seinen Versuchen „Über die Frage eines Koordinationzentrums im Herzventrikel", in denen die Hunde nach Unterbindung einer oder zweier Koronararterien mehrere Stunden bis Tage lebten, hatte Porter die Hunde nur ätherisiert.

4) Heart vol. 4 no. 4. 1913. 
Diese Angaben mögen genügen zum Beweis dafür, dass die Narkotika die Flimmerdisposition des Herzens zu ändern vermögen, und dass hierbei nicht nur die Art des Nárkotikums, sondern auch seine $M$ enge zu berücksichtigen ist.

Ein weiterer Koeffizient, der $\mathrm{Z}$ zu ändern vermag, ist der bei der Thoraxeröffnung jeweilig verschiedene Blutverlust. Da dieser Koeffizient die Ischämiehypothese berührt, wollen wir ihn erst mit dieser besprechen.

Endlich seien hier noch die Nebenverletzungen des Herzens angeführt, deren Bedeutung für die Erklärung des Hkf nach Kav von Tigerstedt und Langendorff so übersehätzt worden ist, dass sie ihnen allein die Schuld geben, wenn nach Kav das Herz flimmerte. Das ist nun zweifellos ein Irrtum, gegen den schon Porter sehr richtig angekämpft hat, dessen Angaben aber nicht genügt haben, um die Nebenverletzungshypothese in Deutschland zu beseitigen, denn sie dominiert hier noch, wie schon eingangs erwähnt wurde.

Als disponierenden, wenn auch im allgemeinen unwesentlichen Koeffizient kann man die bei der Freilegung oder Umstechung einer Koronararterie eintretende Nebenverletzung des Herzens wohl ansehen, und insofern sei sie hier bei Besprechung von $Z$ auch erwähnt.

Als auslösender Koeffizient kommt die Nebenverletzung aber nur als Versuchsfeh] er bei der Operation in Betracht, das heisst dann, wenn das Hkf unmittelbar an die Freilegung, Umstechung, Unterbindung oder Abklemmung der Koronararterie eintritt, wie dies im Gefolge einer mechanischen Reizung der Fall ist. Dass eine Nebenverletzung der Herzwand Flimmern hervorrufen kann, wird niemand leugnen, sagt Porter, und hat damit vollkommen recht; er hätte nur noch hinzufügen sollen, dass, wenn es bei den Vorbereitungen zur Koronarunterbindung oder dieser selbst eintritt,ein Versuchsfehler vorliegt, was er dem Sinne nach, entsprechend seinen weiteren Ausführungen wohl auch meint.

Um die mechanische Verletzung auszuschalten, verschloss er die Öffnung der Koronararterien mit einem Glasstabe von der Aorta aus. Es bedarf aber gar nicht dieser besonderen Methode, um zu zeigen, dass die mechanische Verletzung bei den in Frage stehendeu Eingriffen nicht als auslösender Koeffizient in Betracht kommt, denn aus der Grösse des Intervalles zwischen Setzung der Verletzung und dem Eintritt des Hkf geht schon mit Sicherheit hervor, ob 
letzteres durch die mechanische Reizung ausgelöst wurde oder nicht. Ich babe schon aus diesem Grunde bei meinen Versuchen immer, wie weiter oben bereits erwähnt, auf das Intervall Kav-Hkf geachtet, von denen das kürzeste $1^{1 / 2}$ Minute dauerte, während das $\mathrm{Hkf}$ auf mechanische Reizung hin bekanntlich innerhalb so kurzer Zeit ausgelöst wird wie eine Extrasystole durch einen mechanischen Reiz. Hiermit ist, meines Erachtens, der Widerlegung der Nebenverletzungshypothese schon mehr Zeit gewidmet, als sie verdient, und ich will nur zum Beweise dafür, was für eine geringe Rolle die Nebenverletzung als auslösender Koeffizient spielt, anführen, dass kein einziger meiner Versuche über die Abbindung oder Abklemmung einer Koronararterie durch die mechanische Reizung sein vorzeitiges Ende fand, was auch aus dem oben angeführten kürzesten, von mir beobachteten Intervall Kav-Hkf hervorgeht.

\section{4. Über die auslösenden Koeffizienten des Herzkammerflimmerns nach Koronararterienverschluss.}

Wenn wir die Kammern elektrisch oder mechanisch direkt reizen und unmittelbar an diese Reizung sich anschliessend Hkf auftritt, so ist es klar und leicht zu sagen, welches der auslösende Koeffizient war. Nicht so bei Kav. Tritt nicht unmittelbar im Anschluss an letzteren, wie bei einer mechanischen Reizung, also durch einen Versuchsfehler, das Hkf auf, sondern erst eine bis viele. Minuten nach dem Kav, so ist zwar der Kav der letzte vor dem Eintritt des Hkf zu den übrigen Koeffizienten von uns hinzugefügte Koeffizient und insofern, der üblichen Auffassung des Begriffes Auslösung entsprechend, der auslösende Koeffizient, nicht aber der letzte unmittelbar vor Erscheinen des Hkf wirkende Koeffizient, wie der mechanische oder elektrische Reiz.

Wie wir auch hier wieder sehen, spielt die Grösse des Intervalles Kav-Hkf bei der weiteren Erklärung des. Hkf nach Kav eine grosse Rolle, der gegenüber zum Beispiel die Variationen der Latenzzeit zwischen einem mechanischen oder elektrischen Reiz und dem unmittelbar darauf erscheinenden Hkf verschwindende sind. Die verschiedene Grösse jenes Intervalles ist es auch, die bei der Erklärung des Hkf nach Kav den verschiedenen Autoren Schwierigkeiten bereitet hat, denn einmal ist es relativ sehr kurz, ein anderes Mal unendlich lang, das heisst, es tritt das Hkf nach Kav während der Beobachtungszeit überhaupt nicht auf. Je mehr Zeit seit dem 
Kav verstrichen ist, bevor das Hkf erscheint, desto weniger macht es begreiflicherweise den Findruck, dass das Hkf durch den Kav veranlasst wurde. Demgegenüber müssen wir wiederum darauf hinweisen, dass das Iutervall Kav-Hkf im Vergleich zu dem Intervall zwischen einem zum Beispiel mechanischen Reiz mit dem Hkf überhaupt sehr lang ist, so dass es für die Erklärung nicht viel ändert, ob das Hkf eiue oder erst eine Anzahl Minuten nach Kav auftritt. Zur vollständigen Erklärung des Erscheinens von Hkf nach Kav bedürfte es einer lückenlosen Kenntnis der Vorgänge; die durch den Kav veranlasst werden. Dass der Kav eine Erschwerung bis Aufhebung der arteriellen Blutzufuhr, das heisst eine Ischämie des von der Arterie normalerweise mit Blut versorgten Bezirkes bewirkt, darauf haben bereits die ersten Experimentatoren bingewiesen. Aber schon diese erste Folge des Kav ist durchaus keine etwa konstaut zu erwartende, wie Cohnheim und v. Schulthess-Rechberg glaubten, die die Koronararterien als Endarterien ansahen, vielmehr ist das Herz reich an arteriellen Anastomosen, was in letzter Zeit besonders von Jamin und Merkel sowie von Spalteholz hervorgehoben wurde, so dass die Möglichkeit eines kollateralen Ausgleiches gegeben ist.

Die ganze Frage nach dem Zusammeuhang zwischen dem Kav und dem Hkf bekommt ein etwas anderes Gesicht und rükt damit unsrem Verständnis wesentlich näher, wenn wir einmal auch den ungekehrten Weg gehen und rückwärts anfangend uns fragen, was deun eigentlich das Herzflimmern für eine Erscheinung ist. Darauf können wir heute antworten, dass es die Folge des höchsten Grades einer heterotopen Reizbildung darstellt. Es handelt sich also bei dem Zustandekommen des Hkf nach Kav darum, dass der Kav eine heterotope Reizbildung veranlasst. Mit dieser Erkenutuis befriedigen wir nicht nur unser Bedürfnis nach der Feststellung des letzten, unmittelbar auslösenden Koeffizienten, welches demgemäss die heterotopen Herzreize sind, sondern wir vermögen dadurch auch aus der Reihe der. Vorgänge, die sich zwischen dem Kav und dem Hkf abspielen, einen hervorzuheben, die heterotope Reizbil lung, diekoustant zu erwarten ist und immer eintreten muss, damit es zu Hkf kommt.

Wie schon eingangs hervorgehoben, hatten Cohnheim und v. Schulthess-Rechberg seinerzeit nicht erkanut, dass das Wesentliche und Primäre des "plötzlichen Herzstillstandes" nach Pflüger's Archiv für Physiologie. Bd. 163. 
Kav das Hkf ist, dass also das Wesentliche und Primäre hierbei eine Reizerscheinung ist. Infolgedessen konnten Cohnheim und v. Schulthess-Rechberg anch nicht erklären, wieso infolge der lokalen Schädigung ein er Kammer beide Kammern in ihrer Totalität so plötzlich versagen können, oder mit andren Worten die Erklärung, die sie auf S. 530 für die "totale Lähmung beider Herzventrikel" gaben, war nicht richtig, da sie übersahen, dass die "totale Iähmung beider Herzventrikel" eine sekundäre Erscheinung und erst die Folge der primär eintretenden Reizung ist.

Zur Erklärung des Zusammenhanges zwischen Kav und Hkf haben wir nach diesen Feststellungen also ausfindig zu machen, wie es nach Kav zur heterotopen Reizbildung kommt.

Dazu sei zunächst bemerkt, dass wir heute schon eine ganze Anzahl Koeffizienten kennen, die zur heterotopen Reizbildung zu führen vermögen, was uns das Verständnis der heterotopen Reizbildung nach Kav wesentlich erleichtert.

Ist, wie gesagt, der Kav auch der letzte von uns hinzugefügte Koeffizient, so löst doch dieser Eingriff nicht etwa als mechanischer Reiz, falls nicht ein Versuchsfehler vorliegt, das Hikf aus, sondern es kommt erst nach Ablauf einer verschieden langen Zeit infolge der lokalen Ischämie zur heterotopen Reizbildung, deren höchster Grad dás Flimmern darstellt.

Wir kommen damit zu jener Hypothese, die wir eingangs Anämiehypothese genannt haben, die aber besser als I s $\mathrm{ch}$ ä $\mathrm{m}$ i e h y p o the se zu bezeichnen ist. Gegen sie ist, wie schon angeführt, besonders Tigerstedt und Langend orff aufgetreten, und zwar, wie wir heute sicher sagen können, mit Unrecht. Sie haben folgende Versuche veröffentlicht, die die Berechtigung ihres ablehnenden Standpunktes beweisen sollten.

Tigerstedt hob durch Abklemmung der Vorhöfe beim Hund den ganzen Kreislauf 115-150 Sekunden lang vollständig auf; in keinem einzigen Falle trat das Herzdelirium ein, weder während noch nach der Abklemmung.

Langendorff durchströmte nach seiner bekannten Methode von der Aorta aus durch die Koronargefässe das Herz von Kaninchen, Katzen und Hunden; nachdem die Herzen schlugen, hob er die Blutzufuhr auf; die Blutsperre bewirkte niemals Flimmern.

Gegen diese Versuche soll an sich nichts gesagt werden, nur beweisen sie nicht, wie Tigerstedt meinte, "dass derjenige Herz- 
stillstand, den Cohnheim und v. Schulthess-Rechberg beobachtet haben, nicht durch die Auämie eines umschriebenen Teiles der Herzwand, sondern durch Nebenverletzungen bedingt ist", oder wie Langend orff meinte, dass seine Ergebnisse „in vollständigem Eiuklang mit der Meinung derjenigen stehen, die wie v. Frey, Tigerstedt u. a. den dauernd schädizenden Einfluss der vorübergehenden Aufhebung der Blutzufuhr zum Herzmuskel leugnen und den Grund für die von v. Bezold, Cohnheim usw. erhaltenen Resultate auf Nebenverletzungen beziehen".

„In dieser Beziehung ist es wichtig", fährt Langendorff fort, „dass es mir in einem Falle, am isolierten Kaninchenherzen, bei dem die Blutsperrung genau wie in den übrigen gemacht und keineswegs Wogen herbeigeführt hatte, gelang, durch Zuklemmung der liuken Art. coron. Wogen herbeizuführen. Unmöglich konnte an diesem die mit der Abklemmung verbundene Aufhebung der Blutzufuhr schuld haben." Hier übersieht Langendorff, wie übrigens schon vor ihm auch Tigerstedt bei seiuem Experiment, dass es doch wohl einen Untersehied geben kann, ob man eine lokale oder eine allgemeine Ischämisierung des Herzens bewirkt, wie in ibren Versuchen, die durchaus nicht widerlegen, dass die Ischämie eines umschriebenen Teiles der Herzwand zu Flimmern fübren kaun.

Wenn auch Frey 1891 meinte, "dass die Anämie des Herzmuskels nach den vorliegenden physiologischen Erfahrungen eine relativ ungefährliche Erscheinung ist", so war er doch später (1894), in Anschluss an die Fxperimente von Porter, wie erwähnt, auf dem richtigen Wege, als er sagte: „Entwerler muss zur Anämie des abgesperrten Herzteiles noch etwas hiuzukommen, wodurch der Stillstand herbeigeführt wird, oder es ist neben der Grösse auch der Ort der Schädigung von besonderer Bedeutung."

Gewiss bewirkt nicht jede lokale Ischämie der Kammern Hkf, wohl aber unter der Mitwirkung der übrigen hierzu erforderlichen Koeffizienten, die our nicht in allen Versuchen immer mitwirken oder denen andere Umstäude entgegenwirken. Wir habeu von den Koeffizienten bisher wesentlich nur die Grösse der Arterie, die Funktion des von ihr versorgten Herzbezirkes und die Disposition der Herzkammern besprochen; aber es kommen gewiss noch andere Koeffizienten in Betracht. Der auslöseude Koeffizient des Hkf, weun auch uicht der letzte, ist sicher die durch den Kav bewirkte lokale 
Ischämie, welche, je rach den Umständen, eine grössere oder kleinere, dauerude oder voräbergehende ist; nur diesem Koeffizienten kaun die Auslösung des uach eiuiger Zeit auftruteuden Hlif zugeschrieben werden (falls nicht, wie sihon öfter erwähut, beim Verschluss der Arterie infolge eines Versuchsfehlers das Herz durch direkt mechanische Reizung unmittelbar im Anschluss an den Eingriff zum Flimmern gebracht wurile), denn alle auleren mit dem Finoriff gegebenen uud je nach Gesehick und Glück verschieden grossen Nebeuverletzungen des Herzens uud seiner Nerven köuneu höchstens die Di-position der Herzkammern etwas ändern, sie bewirken aber, wie schon Porter hervorgehoben hat, dem ich hier vollkommen beistimme, nieht das Flimmeru. Wenn es nun auch, wie erwähnt, einen Uıterschied marht, ob man eine lokale oder allyemeine Isrhämierung des Herzens hewirkt, so hahen dorh heide das Gemeinsame, dass sie die Disposition zum Flimmern zu erhöhen vermö̈ren, falls sie es nicht auslösen, deun, wie sihou Porter auführt, tritt auch nach Verblutung leiıht Flimmern auf, worin ich ihm auf Grund reicher Erfahrung uur beistimmen kaun.

Wir kömnen auf Grund der vorliegenden Erfahrungen die Beziehuug zwischen dem Kav und $\mathrm{Hkf}$ zunächst folgendermaassen präzisieren :

Kommt es infolge Koronararterienverschluss zu Herzkammerflimmern, so ist die lokale Ischämie einer seiner Koeffizienten.

Dies ist der Borlen, auf dem wir uns bei der Analyse der weiteren Folgen des zu Hkf führenden Kav stellen müssen. Bis hierher war sie noch relativ leicht; aber sie wird, wie bei allem lebendigen Geschehen, um so schwieriger, je tiefer wir einzudingen versuchen.

Schon Cohnheim versuchte dies und frug sich: „Ist nun aber die Anäın e als solche oder, um es präriser auszudrücken, der Mangel an sauerstoff haltigem Blut die eigentliche Ursache des ganzen Vorganges?" Nach Verneinung dieser Frage kam er zu der Vermutung, "dass hier die Wirkung einer positiven, für das Herz direkt schädli.hen Substanz, eiues wirklichen Herzgiftes vorliegt, das sich während des Verschlusses des Kranzarterienastes gebildet hat". Als Uuterstützung für diese Vermutung führt er an: „Eine gewisse Ähnlichkeit unserer typischen Kurven mit beispielsweise der von der Kalivergiftung wird niemand verkennen." 
Cohnheim war hier insofern auf dem richtigen Wege, als er die von ihm beobaclitete schädliche Wirkung des Kav irgeudwelchen Stoffen zuschrieb. Speziell meinte er, dass es sich um Substanzen handle, die "unter dem Finflusse der Herıkontraktionen im Stoffwechsel des Herzmuskels kontinuierlich produziert werden, welche für Muskel- und Nerventätigkeit direkt schädlich sind". Er irrte jedoch iusofern, als er glaubte, dass die in dem umschriebenen Bezirke gebildeten Substauzen eine totale Lähmung der beiden Herzventrikel hewirken; da in jenem Bezirke der Blutstrom nach seiner eigenen Angabe unterbrochen ist, können jene Substanzen wohl eine lok a le Vergiftung, nicht aber "eine totale Lähmung beider Herzventrikel" bewirken. Jene Auffassung hängt mit dem schon erwähnten Umstande zusammen, dass er nicht erkannte, dass das Primäre das Flimmern ist, das sehr wohl durch eine lokale Vergiftung hervorgerufen werden kann.

In der Tat muss die Folge des Kav, wenn er zu einer lokalen Ischämie führt, eine lokale endogene Vergiftung sein.

Fine weitere Frage ist es nun, welche Stoffe es siud, die zum Auftreten des Hkf heitragen können, ob wesentlich ein Stoff als auslösender bezeichnet werten kann.

Die vielfache Beschäftioung mit der $L$ angend orff'schen Methode der küustlichen Durchströmung des Säugetierherzens brarhte mich auf den Gedanken, die Versuche üher die Folgen des Kav auch an Herzen vorzunehmen, die nur mit Ringer'scher Lösung gespeist werden. Würle hei diesen Herzen nach Verschluss einer Koronararterie ebenfalls Flimmern auftreten, dann war damit entschied"'n, dass die übrigen im Blute, nicht aber in der Ringer'schen Lösung vorhandenen Stoffe notweudigerweise nichts damit zu tun haben.

Meine an Huudeherzen angestellten Versuche hahen nun in der Tat ergeben, dass auch, an den nur mit Ringer'scher Lösung gespeisten Hundeherzen derVerschluss einer grösseren Koronararterie Hkf herbeizuführenvermag.

Diese Versuche liefern durch die Art und Weise, wie ich sie anstellte, gleichzeitig auch einen weiteren Beweis gegen die Nebenverletzungshypothese, wenn es uberhaupt eines soleh n noch bedurfte. Ich habe nämlich bei diesen Versuchen die Arterie, zum Beispiel die Coronaria dextra, vor der Wiederbelebung des verbluteten Herzens präpariert; so kann man auf das Peinlichste alles für die später, nach erfolgter Wiederbelebung des Herzens vorzunehmenden Abklemmung oder Abbindung der Arterie vorbereiten. 
Die Tatsache, dass auch an den nur mit Ringer'scher Lösung gespeisten Hundeherzen Hkf nach Kav auftritt, vereinfacht die Erforschung der an der lokalen Vergiftung beteiligten Stoffe sehr wesentlich. Wir sehen hier das Flimmern auftreten als die Folgeerseheinung der lokalen Absperrung einer sauerst offhaltigen Salzlösung.

Die Folge der lokalen Absperrung der Speisungsflüssigkeit ist mit Bezug auf den Stoffwechsel eine zweifache: das Fehlen neuer Zufuhrstoffe und die Anhäufung der Abfuhrstoffe. Würde es sich nur um die Abschneidung der Zufuhr bei unbehinderter Abfuhr der Stoffwechselprodukte handeln, so würde der betroffene Herzabschnitt eine Lähmung erfahren. In Wirklichkeit sehen wir aber, dass an dem betroffenen Herzabschnitt auch Erregungs erscheinungen auftreten und diese sind wohl durch die Anhäufung von Abfuhrst of $f$ en veranlasst. Wenn wir auch über die letzteren noch durchaus nicht befriedigend unterrichtet sind, so kennen wir doch besonders einen dieser Stoffe und seine Wirkungsweise, das ist die Kohlensäure. Auf Grund der bis jetzt vorliegenden Erfahrungen glaube ich, dass vielleicht die Kohlensäure ein wesentlicher Koeffizient für das nach Kav auftretende Hkf anzusehen ist." Dafür sprechen folgende Tatsachen:

1. Es ist bekannt und von mir oft genug beobachtet worden, dass bei Erstickung des Tieres die Kammern lejeht ius Flimmern geraten, dass also Erstickung, je nach seinem Grarle, ein auslösender oder ein das Auftreten von Hkf begünstigender Koeffizient ist.

2. Beobachtet man zum Beispiel nach Unterbindung der rechten Coronaria den von ihr versorgten Herzbezirk, so sieht man ihn zunächst abblassen, alsbald aber eine livide Färbung annehmen; es tritt eine lokale Erstickung ein.

3. Aus Versuchen von $\mathrm{R}$ Magnus aus dem Jahre 1902, die er zu anderen $Z$ wwecken vorgenommen hat, geht hervor, dass die Durchströmung der Koronargefässe mit Kohlensäure die Kammern des isolierten Katzenherzens zum Flimmern bringt. Es ist demnach möglich, dass ein w esentlicher Koeffizient des infolge lokaler Ischämie auftretenden Herzkammerflimmern die Kohlensäure ist.

Welche andere Stoffe ausser der Kohlensäure an dieser lokalen endogenen Vergiftung sich noch beteiligen und zum Auftreten von 
Hkf beitragen, wird Sache weiterer Untersuchungen sein, wobei an die Milchsäure, Phosphorsäure usw. wie überhaupt an die Wasserstoffionenkouzentration zu deuken sein wird. Damit, dass ich die Kohlensäure als einen wesentlichen Koeffizienten für Hkf nach Kav ansehe, ist nicht gesagt, dass die Kohlensäure unter allen Umständen Hkf bewirkt. So konnten wir in den Versuchen, die ich im Jahre 1903 mit E. Gross über den Einfluss der Kohlensäure auf das mit Ringer'scher Lösung durchströmte Hundeherz machte, nur Lähmungserscheinungen, aber keine Erregungerscheinungen beobachten, wenn wir den Kohlensäuregehalt der Lösung erhöhten. Das ist jedoch kein Beweis gegen die oben geäusserte Auschauung, wenn es mir auch Veranlassung gibt, nach dem Kriege die Versuche zu wiederholen. Es ist bekannt, dass die Kohlensäure erregend wirkt, und auch dass diese Wirkung im allgemeinen weniger leicht zu zeigen ist als die lähmende. Wovon das abhängt, wollen wir hier nicht weiter erörtern. Es sei nur noch darauf hingewiesen, dass an der von $\mathrm{M} \mathrm{ag} \mathrm{n}$ u s veröffentlichten Kurve (Fig. 7) das Flimmern erst auftritt, nachdem schon einige Zeit hindurch Lähmungserscheinungen vorausgegangen siud. Diese Tatsache erinnert an die von mir ${ }^{1}$ ) kürzlich hervorgehobene Beobachtung, dass schon vor dem durch $\mathrm{KCl}$ bewirkten Kammerflimmeru die kontraktionsschwächende Kaliwirkung bemerkbar ist. Mit diesen Beobachtungen harmoniert nun auch, dass an dem von der lokalen Ischämie betroffenen Kammerbezirk vor dem Auftreten des Flimmerns eine ausgesprochene Kontraktionsschwäche zu bemerken ist, die ich schon wiederholt graphisch aufgenommen habe.

Wie das $\mathrm{KCl}$ wirkt hier die lokale Ischämie und die Kohlensäure (wenigstens in der angedeuteten Weise und Menge) lähmend auf die Kontraktilität, erregend auf heterotope Reizbildungsstellen. Wir haben uns in dieser Mitteilung, wie eingangs erwähnt, auf die erregende Wirkung des Kav beschränkt, da sie das Wesentliche ist für die Tatsache, dass eine lo kale Ischämie einer Kammer beide Kammern in ihrer Totalität plötzlich zum Versagen bringt, fügen hier aber jenen Koeffekt des Kav, die lokale Lähmung, ergänzend hinzu, da die Herabsetzung der Kontraktilität das Auftreten des Hkf bekanntlich begüustigt.

1) Pflüger's Arch. Bd. 161 S. 544. 1915. 


\section{Sehlussbemerkungen.}

Aus den vorausgehenden Ausführungen dürfte zur Genüge hervorgehen, warum die verschiedenen Autoren über die Folven des Kav so verschiedecer Meinung sind. Es ist das eben ein Eingriff, dessen Erfolg sehr verschieden sein kaun. Das eine Mal Auftreten von Hkf nạch $1^{1 / 2}$ Minuten, ein anderes Mal erst nach vielen Minuten, ein drittes Mal nur Infarkte oder selbst diese nicht, wie in dem bekannten Falle von Pagenstecher ${ }^{1}$ ), der den R. descendens der linken A. coronaria beim Meuschen hoch über der Herzspitze unterband und 5 Tage nach der Unterbindung, als der Patient eiuer Perikarditis erlag, die von der unterbuudenen Arterie versorgte Muskulatur gegenüber der übrigen Herzmuskulatur nicht verändert fand.

Wie wir gesehen haben, hängt der uns hier interessierende Frfolg des Kav, das Hkf, von einer Anzahl von Koeffizieuten ab, von denen wir den Kav, die lokale Ischämie, die lokale Vergiftung durch Behinderung der Zu- und Abfuhr der Stoffwechselstoffe, wahrscheinlich wesentlich die Kohlensäure und schliesslich die heterotopen Herzreize nacheinander als auslösende, die Grösse der Arterie, die Funktion des von ihr versorgten Bezirkes, die Narkose, die Blutung, die Nebenverletzungen usw. als disponierende Koeffizienten bezeichuen können.

Fassen wir die Summe der disponierenden Koeffizienten unter dem üblichen Ausdruck Disposition (D) zusammen und stellen ihr die Auslösung (A) gegenüber, so kann in dem Verhältnis A:D bekanntlich A um so geringer sein, je grösser D ist und umgekehrt. Dementsprechend kann die lokale Ischämie desto kleiner sein, je grösser D ist, und wird um so grösser sein müssen, je geringer D ist. Diese auf anderen Genieten heute sehr geläufige Beziehung von $A: D$ ist bei der Beurteilung der Versuche über die Folgen des Kav nicht entsprechend beachtet worden, obwohl seine Beachtung gerade bei der uns hier in dieser Mitteilung hauptsächlich interessierenden Folgeerscheiuung, dem Hkf, besouders nötig ist.

Mein Interesse an dem durch Kav hervorgerufenen Hkf hängt auch mit dem durch Hkf bedingten plötzlichen Torle zusammen. Der Umstand, dass weder Cohnheim und v. Schulthess-Rechberg noch Porter erkannten, dass das Wesentliche und Primäre

1) Deutsch. med. Wochensch. 1901 H. 4 S. 56. 
des plötzlichen Versagens der beilen Kammern nach Kav das Hkf ist, trug gewiss mit dazu bei, der schon von M c.William 1887 im Brit. med. Journ. ausgesprncheuen Vermutung, Jass plöt»liche Todesfälle bein Menschen durch Eiutritt fibrillärer Kontraktionen des Herzmuskels bedingt sein könuten, nicht die ihr zukommende Beachtung zu scheuken.

Noch hiuderlicher war aber in verschiedener Beziehung die von Tigerstedt und Langendorff vertretene und vielfach angenommene Anschauung, dass das Hkf nach Kav auf Nebenverletzungen beruhe.

\section{Zusammenfassung.}

Kav hat nur im Verein mit anderen Koeffizienten Hkf zur Folge; letzteres kann daher nicht die notwendige Folge nur des ersteren sein.

Soweit es bis jetzt bekannt ist, gehören zu diesen Koeffizienten die Grösse der Arterie, die Funktion des von ihr versorgten Bezirkes, die Narkose, die Blutung, die Nebenverletzungen (disponierende Koeffizienten).

Das Hkf nach Kav nur auf die Nebenverletzungen zu beziehen, wie Langendorff und Tigerstedt es taten, ist unzutreffend. Tritt unmittelbar im Anschluss an eine Verletzung des Herzens, wie eine Extrasystole auf einen mechavischen Reiz, Flimmern auf, so ist dies bei den Versuchen über die Folgen des Kav ein Versuchsfehler. Das kürzeste an 20 Hundeherzen von mir beobachtete Intervall zwischen Kav und dem Beginn des Hkf betrug 11/2 Minuten.

Zusammenfassend kann man sagen, dass es von dem jeweiligen Verhältnis des Grades der Disposition (D) der Herzkammern zur Grösse der Auslösung (A) abhängt, ob und wie rasch nach Kav Hkf eintritt, mit anderen Worten wie gross das Intervall Kav-Hkf ist.

Als auslösende Koeffizienten kommen nacheinander in Betracht: der Kav, die lokale Ischämie, die lokale Vergiftung, vielleicht als wesentlich die Kohlensäure und schliesslich die heterotopen Herzreize.

Ein das Flimmern unterstützender Koeffizient ist die als Koeffekt des Kav auftretende lokale Herabsetzung der Kontraktilität.

Auch an nur mit Ringer'scher Lösung gespeisten Hundeherzen vermag der Verschluss einer grösseren Koronararterie Hkf herbeizuführen. 
26 H. E. Hering: Über die Koeffizienten, die Herzkammerflimmern bewirken.

Kommt es nach Kav zu Hkf, so geht diesem nicht, wie Cohnheim und v. Schulthess-Rechberg und auch Porter meinten, ein Stillstand des Herzens voraus.

Das plötzliche Versagen beider Kammern nach Kav beruht auf dem lokal ausgelösten Flimmern beider Kammern, durch welches sich auch Fälle von ganz plötzlichem Herztod beim Menschen erklären lassen.

Nachtrag: Aus einer Mitteilung von E. Louis Backman ${ }^{1}$ ) über „Die Wirkung der Milchsäure auf das isolierte und überlebende Säugetierherz" geht hervor, dass die Milchsäure "selbst in den niedrigsten Konzentrationsgraden" lähmend auf die Kontraktilität, in etwas höheren Konzentrationsgraden, als sie normalerweise im Blut vorkommen, erregend auf die Reizbildung wirkt. Demnach könnte, wie schon weiter oben erwähnt, auch die Milchsäure als Koeffizient für das Herzkammerflimmern nach Koronararterienversehluss mit in Betracht kommen; da sich die vorliegenden Angaben über die erregende Wirkung der Milchsäure auf die nomotope Reizbildungsstelle bezieht, wird noch festzustellen sein, ob das gleiche auch für die heterotopen Reizbildungsstellen gilt. Wenn dies der Fall wäre, dann würde sich die Milchsäure ähnlich verhalten wie die Kohlensäure und es wäre dann noch zu untersuchen, wie auch schon oben erwähnt, ob die Wasserstoffionenkonzentration massgebend ist, und ob, wenn dies der Fall ist, eine der Säuren durch ihre Quantität einen besonderen Eiufluss besitzt, oder ob die Qualität einer Säure in Betracht kommt.

1) Skandinav. Arch, f. Physiol. Bd, 20 S. 162. 1908. 